\title{
A CHARACTERIZATION OF LOCALLY CONNECTED CONTINUA BY HYPERSPACE RETRACTIONS ${ }^{1}$
}

\author{
SAM B. NADLER, JR.
}

\begin{abstract}
Let $X$ be a (metric) continuum. It is shown that $X$ is locally connected if and only if there is special type of retraction from $2^{X}$ onto $C(X)$, where $2^{X}$ [resp., $C(X)$ ] is the space of all nonempty compact subsets [resp., subcontinua] of $X$ with the Hausdorff metric. Also, necessary and sufficient conditions are given for the "continuity of balls".
\end{abstract}

1. Introduction. Throughout this paper, $X$ denotes a continuum (i.e., a compact connected metric space containing more than one point),

$$
2^{X}=\{A \subset X: A \text { is nonempty and compact }\}
$$

and

$$
C(X)=\left\{A \in 2^{X}: A \text { is connected }\right\}
$$

where $2^{X}$ and $C(X)$ have the topology obtained from the Hausdorff metric [see (2.6)]. Goodykoontz [4] has characterized dendrites within the class of hereditarily unicoherent continua as follows:

(1.1) TheOREM [4, TheOREM 1]. Let $X$ be an hereditarily unicoherent continuum. For each $A \in 2^{X}$, let $I(A)$ denote the unique subcontinuum of $X$ irreducible about $A$. Then, $I$ is continuous [as a function from $2^{X}$ to $\left.C(X)\right]$ if and only if $X$ is a dendrite.

Let $X$ be an hereditarily unicoherent continuum. Note that $I$, defined in (1.1), has the following properties:

(i) $I(A) \supset A$ for each $A \in 2^{X}$ and

(ii) $I(A)=A$ if and only if $A \in C(X)$.

Thus, by (1.1) and (ii), $X$ is a dendrite if and only if $I$ is a retraction from $2^{X}$ onto $C(X)$ [a retraction is a continuous function which is the identity on its range]. The main purpose of this paper is to characterize locally connected continua from among all continua by means of the existence of retractions having properties similar to those of I. Specifically, I will prove the following result:

Received by the editors March 17, 1977.

AMS (MOS) subject classifications (1970). Primary 54B20; Secondary 54C15, 54F65.

Key words and phrases. Convex metric, dendrite, Hausdorff metric, hereditarily unicoherent continuum, locally connected continuum, retraction, selection.

'This paper was written, while the author was on a visiting position at the University of Kentucky, Lexington, Kentucky. 
(1.2) MAIN TheORem. Let $X$ be a continuum.

(1.2.1) If $X$ is locally connected, then there is a retraction $r: 2^{X} \stackrel{\text { onto }}{\rightarrow} C(X)$ such that $r(A) \supset A$ for each $A \in 2^{X}$ [see (5.1)].

(1.2.2) If there is a retraction $g: 2^{X} \stackrel{\text { onto }}{\rightarrow} C(X)$ such that $g(A) \supset A$ or $g(A) \subset$ $A$ for each $A \in 2^{X}$, then $X$ is locally connected.

From the discussion above, we see that (1.2) is in a sense analogous to and an extension of (1.1). Other reasons for interest in (1.2) will be discussed in $\$ 6$.

In proving (1.2.1), I will use a convex metric on $X$ to construct $r$. A number of results are given in $\S \S 3$ and 4 to aid in the definition of $r$ and the proof of its continuity. Many of these results are specialized and technical in nature. However, (3.3) and (3.4) would seem to be of some independent interest. They determine when the closed balls defined in (2.2) and (2.4) vary continuously with their centers and radii. In $\S 5, r$ is defined and the proof of (1.2) is completed.

The author is indebted to Carl Eberhart for some conversations concerning the material in this paper.

2. Notation and basic facts. Let $(X, d)$ denote a continuum with metric $d$. Let $\varepsilon \geqslant 0$. If $a \in X$, then

$$
B_{d}(\varepsilon, a)=\{x \in X: d(a, x)<\varepsilon\}
$$

and

$$
C_{d}(\varepsilon, a)=\{x \in X: d(a, x) \leqslant \varepsilon\} .
$$

More generally: If $A \in 2^{X}$ and $\varepsilon \geqslant 0$, then

$$
N_{d}(\varepsilon, A)=\cup\left\{B_{d}(\varepsilon, a): a \in A\right\}
$$

and

$$
K_{d}(\varepsilon, A)=\cup\left\{C_{d}(\varepsilon, a): a \in A\right\} .
$$

As is well known and easy to prove,

$$
\begin{aligned}
& K_{d}(\varepsilon, A) \text { is a closed and, therefore, } \\
& \text { compact subset of } X \text { for each } A \in 2^{X} .
\end{aligned}
$$

Also note that: For any $a \in X, N_{d}(\varepsilon,\{a\})=B_{d}(\varepsilon, a)$ and $K_{d}(\varepsilon,\{a\})=$ $C_{d}(\varepsilon, a)$; for any $A \in 2^{X}, N_{d}(0, A)=\varnothing\left[=\right.$ empty set] and $K_{d}(0, A)=A$. Recall that the Hausdorff metric $H_{d}$ for $2^{X}$ is defined by the following formula: For any $A, B \in 2^{X}$,

$$
H_{d}(A, B)=\inf \left\{\varepsilon>0: A \subset N_{d}(\varepsilon, B) \text { and } B \subset N_{d}(\varepsilon, A)\right\} \text {. }
$$

The topology for $2^{X}$ may be described as follows: A sequence $\left\{A_{i}\right\}_{i=1}^{\infty}$ in $2^{X}$ converges with respect to $H_{d}$ to $A_{0} \in 2^{X}$, written $A_{i} \rightarrow A_{0}$ as $i \rightarrow \infty$, if and only if

$$
\lim \inf A_{i}=A_{0}=\lim \sup A_{i}
$$


where lim inf and lim sup are as defined in [13, p. 10]. The spaces $2^{X}$ and $C(X)$ are each compact.

A metric $\rho$ for a set $S$ is said to be convex [9] provided that given $x, y \in S$, there exists $z \in S$ such that

$$
\rho(x, z)=\rho(y, z)=\rho(x, y) / 2 .
$$

The following fact is well-known and easy to prove (take successive midpoints and then extend from the dense set):

(2.7) THEOREM [9]. Let $(X, \rho)$ be a continuum with a convex metric $\rho$. Then, for any $x, y \in X$, there exists a subset $\gamma$ of $X$ such that $x, y \in \gamma$ and $\gamma$ is isometric to the interval $[0, \rho(x, y)]$; clearly $\gamma=\{x\}=\{y\}$ if $x=y$ and, if $x \neq y, \gamma$ is an arc with end points $x$ and $y$.

Using (2.7), it is easy to prove the following result-the symbol cl denotes closure.

(2.8) TheOrem [1, TheOrem 2]. Let $(X, \rho)$ be a continuum with a convex metric $\rho$. Then, for any $a \in X$ and $\varepsilon>0$,

$$
\operatorname{cl}\left[B_{\rho}(\varepsilon, a)\right]=C_{\rho}(\varepsilon, a) .
$$

The following example is worth noting in view of the results in (3.3) and (3.4).

(2.9) Example. Let $S^{1}$ be the unit circle in the plane $R^{2}$,

$$
S^{1}=\left\{(x, y) \in R^{2}: x^{2}+y^{2}=1\right\},
$$

with the metric $d_{1}$ obtained from the usual Euclidean norm $\|$, $\|(x, y)\|=\left(x^{2}+y^{2}\right)^{1 / 2}$. Then, $\left(S^{1}, d_{1}\right)$ satisfies the conclusion of (2.8) even though $d_{1}$ is not a convex metric for $S^{1}$. On the other hand, let

$$
X=\left\{(\cos [\theta], \sin [\theta]) \in R^{2}: 0 \leqslant \theta \leqslant(3 \pi) / 2\right\}
$$

and let $d_{2}$ be the metric for $X$ obtained from \|\| . Then, $\left(X, d_{2}\right)$ does not satisfy the conclusion of $(2.8)$ since, for $a=(0,-1)$ and $x=(+1,0)$, $x \in C_{d_{2}}(\sqrt{2}, a)$ and $x \notin \mathrm{cl}\left[B_{d_{2}}(\sqrt{2}, a)\right]$.

The symbol $\times$ will denote cartesian product. Complementation of sets will be denoted using the symbol -.

Let $X$ be a continuum. For each $n=1,2, \ldots$, let

$$
F_{n}(X)=\left\{A \in 2^{X}: A \text { has at most } n \text { points }\right\} .
$$

The following lemma will be used in the proof of (1.2.2).

(2.11) Lemma. For any continuum $X, 2^{X}-C(X)$ is connected.

Proof. For each $n \geqslant 3$, let $X_{n}=X \times X \times \cdots \times X n$ times,

$$
\Delta_{n}=\left\{\left(x_{1}, x_{2}, \ldots, x_{n}\right) \in X_{n}: x_{i}=x_{j} \text { for all } i \text { and } j\right\},
$$

and let $f_{n}: X_{n}-\Delta_{n} \stackrel{\text { onto }}{\rightarrow} F_{n}(X)-F_{1}(X)$ be given by

$$
f_{n}\left(x_{1}, x_{2}, \ldots, x_{n}\right)=\left\{x_{1}, x_{2}, \ldots, x_{n}\right\} \text {. }
$$


for each $\left(x_{1}, x_{2}, \ldots, x_{n}\right) \in\left[X_{n}-\Delta_{n}\right]$. For each $n \geqslant 3$, it is easy to see that $X_{n}-\Delta_{n}$ is connected and that $f_{n}$ is continuous. Hence, $F_{n}(X)-F_{1}(X)$ is connected and, therefore,

$$
Z=\bigcup_{n=3}^{\infty}\left[F_{n}(X)-F_{1}(X)\right]
$$

is a connected subset of $2^{X}-C(X)$. Also, as is easy to prove, $Z$ is a dense subset of $2^{X}-C(X)$. The lemma now follows.

3. Continuous variance of balls. In general, for a continuum $(X, d)$, there can exist a sequence $\left\{a_{n}\right\}_{n=1}^{\infty}$ of points of $X$ and a sequence $\left\{t_{n}\right\}_{n=1}^{\infty}$ of positive numbers such that $a_{n} \rightarrow a_{0} \in X$ as $n \rightarrow \infty, t_{n} \rightarrow t_{0} \in[0,+\infty)$ as $n \rightarrow \infty$, but $C_{d}\left(t_{n}, a_{n}\right) \nrightarrow C_{d}\left(t_{0}, a_{0}\right)$ as $n \rightarrow \infty$ [see (3.3) and the continuum $\left(X, d_{2}\right)$ in (2.9)]. Thus, the function $\varphi_{d}$ defined below need not be continuous.

(3.1) Definition. Let $(X, d)$ be a continuum. Define $\varphi_{d}:[0,+\infty) \times 2^{X} \rightarrow$ $2^{X}$ by $\varphi_{d}(t, A)=K_{d}(t, A)$ for each $t \in[0,+\infty)$ and each $A \in 2^{X}$.

We will determine when $\varphi_{d}$ is continuous. First, we prove the following lemma.

(3.2) Lemma. Let $(X, d)$ be a continuum. For each $j=0,1,2, \ldots$, let $t_{j}>0$ and let $A_{j} \in 2^{X}$. If $t_{j} \rightarrow t_{0}$ and $A_{j} \rightarrow A_{0}$ as $j \rightarrow \infty$, then

$$
\operatorname{cl}\left[N_{d}\left(t_{0}, A_{0}\right)\right] \subset \lim \inf \operatorname{cl}\left[N_{d}\left(t_{j}, A_{j}\right)\right] \text {. }
$$

Proof. Since $\lim \inf \operatorname{cl}\left[N_{d}\left(t_{j}, A_{j}\right)\right]$ is a closed subset of $X[13$, p. 10], it suffices to show that

$$
N_{d}\left(t_{0}, A_{0}\right) \subset \lim \inf \mathrm{cl}\left[N_{d}\left(t_{j}, A_{j}\right)\right] .
$$

To prove (\#), let $x \in N_{d}\left(t_{0}, A_{0}\right)$. Then, there exists $a_{0} \in A_{0}$ such that $d\left(x, a_{0}\right)<t_{0}$. Choose and fix $\eta>0$ such that $d\left(x, a_{0}\right)+\eta<t_{0}$. Then, since $t_{j} \rightarrow t_{0}$ and $A_{j} \rightarrow A_{0}$ as $j \rightarrow \infty$, there exists a natural number $J$ such that

(1) $j \geqslant J$ implies $d\left(x, a_{0}\right)+\eta<t_{j}$ and

(2) $j \geqslant J$ implies $H_{d}\left(A_{0}, A_{j}\right)<\eta$. By (2) [and (2.6)], $j \geqslant J$ implies $A_{0} \subset$ $N_{d}\left(\eta, A_{j}\right)$. Hence, for each $j \geqslant J$, there exists $a_{j} \in A_{j}$ such that $d\left(a_{0}, a_{j}\right)<\eta$. Thus,

(3) $j \geqslant J$ implies $d\left(x, a_{j}\right) \leqslant d\left(x, a_{0}\right)+d\left(a_{0}, a_{j}\right)<d\left(x, a_{0}\right)+\eta$. By (1) and (3), $j \geqslant J$ implies $d\left(x, a_{j}\right)<t_{j}$; hence,

(4) $j \geqslant J$ implies $x \in N_{d}\left(t_{j}, A_{j}\right)$. Clearly, by (4), $x \in \lim \inf \operatorname{cl}\left[N_{d}\left(t_{j}, A_{j}\right)\right]$. This proves (\#) and, therefore, completes the proof of (3.2).

The following theorem gives necessary and sufficient conditions in order that $\varphi_{d}$ be continuous.

(3.3) Theorem. Let $(X, d)$ be a continuum and let $\varphi_{d}$ be as defined in (3.1). Then, the following three statements are equivalent:

(3.3.1) $\varphi_{d}$ is continuous;

(3.3.2) $\operatorname{cl}\left[B_{d}(t, a)\right]=C_{d}(t, a)$ for each $t>0$ and each $a \in X$;

(3.3.3) $\operatorname{cl}\left[N_{d}(t, A)\right]=K_{d}(t, A)$ for each $t>0$ and each $A \in 2^{X}$. 
Proof. Assume (3.3.1) holds. Let $t>0$ and let $a \in X$. Choose a sequence $\left\{t_{n}\right\}_{n=1}^{\infty}$ such that $t_{n} \rightarrow t$ as $n \rightarrow \infty$ and $0<t_{n}<t$ for each $n=1,2, \ldots$ Then, by (3.3.1),

$$
\varphi_{d}\left(t_{n},\{a\}\right) \rightarrow \varphi_{d}(t,\{a\}) \text { as } n \rightarrow \infty .
$$

Let $x \in C_{d}(t, a)$. Since $C_{d}(t, a)=\varphi_{d}(t,\{a\})$, it follows easily from (1) that there exists a sequence $\left\{x_{n}\right\}_{n=1}^{\infty}$ such that $x_{n} \rightarrow x$ as $n \rightarrow \infty$ and such that $x_{n} \in \varphi_{d}\left(t_{n},\{a\}\right)$ for each $n=1,2, \ldots$ Since $x_{n} \in \varphi_{d}\left(t_{n},\{a\}\right)$ and $t_{n}<t$ for each $n=1,2, \ldots, x_{n} \in B_{d}(t, a)$ for each $n=1,2, \ldots$ Therefore, since $x_{n} \rightarrow x$ as $n \rightarrow \infty, x \in \operatorname{cl}\left[B_{d}(t, a)\right]$. Thus, since $x \in C_{d}(t, a)$ was arbitrary, we have proved

$$
C_{d}(t, a) \subset \operatorname{cl}\left[B_{d}(t, a)\right] .
$$

Clearly, $\operatorname{cl}\left[B_{d}(t, a)\right] \subset C_{d}(t, a)$ and, thus, $C_{d}(t, a)=\operatorname{cl}\left[B_{d}(t, a)\right]$. Therefore, since $t>0$ and $a \in X$ were arbitrary, we have proved (3.3.1) implies (3.3.2). Next we prove (3.3.2) implies (3.3.3). To do this, assume (3.3.2) holds. Let $t>0$ and let $A \in 2^{X}$. Let $x \in K_{d}(t, A)$. Then, there exists $a \in A$ such that $d(x, a) \leqslant t$. Hence, $x \in C_{d}(t, a)$ and so, by (3.3.2), $x \in \operatorname{cl}\left[B_{d}(t, a)\right]$. Since $a \in A, \quad B_{d}(t, a) \subset N_{d}(t, A)$. Thus, since $x \in \operatorname{cl}\left[B_{d}(t, a)\right], \quad x \in \operatorname{cl}\left[N_{d}(t, a)\right]$. Therefore, since $x \in K_{d}(t, A)$ was arbitrary, we have proved

$$
K_{d}(t, A) \subset \operatorname{cl}\left[N_{d}(t, A)\right] .
$$

Clearly, $\operatorname{cl}\left[N_{d}(t, A)\right] \subset K_{d}(t, A)$ and, thus,

$$
K_{d}(t, A)=\operatorname{cl}\left[N_{d}(t, A)\right] .
$$

Therefore, since $t>0$ and $A \in 2^{X}$ were arbitrary, we have proved (3.3.2) implies (3.3.3). Next we prove (3.3.3) implies (3.3.1). To do this, assume (3.3.3) holds. To show that $\varphi_{d}$ is continuous, let $\left(t_{0}, A_{0}\right) \in[0,+\infty) \times 2^{X}$ and let $\left\{\left(t_{n}, A_{n}\right)\right\}_{n=1}^{\infty}$ be a sequence converging to $[0,+\infty) \times 2^{X}$ to $\left(t_{0}, A_{0}\right)$. Since $2^{X}$ is compact, $\left\{\varphi_{d}\left(t_{n}, A_{n}\right)\right\}_{n=1}^{\infty}$ has a convergent subsequence, denoted by $\left\{\varphi_{d}\left(t_{j}, A_{j}\right)\right\}_{j=1}^{\infty}$, with limit denoted by $L$, i.e.,

$$
\varphi_{d}\left(t_{j}, A_{j}\right) \rightarrow L \text { as } j \rightarrow \infty \text {. }
$$

We will show, from which it follows that $\varphi_{d}$ is continuous at $\left(t_{0}, A_{0}\right)$, that

$$
L=\varphi_{d}\left(t_{0}, A_{0}\right) \text {. }
$$

Since $(*)$ is clear if $t_{0}=0$, assume without loss of generality that $t_{j}>0$ for each $j=0,1,2, \ldots$ To prove (*), first note that $L=\lim \inf \varphi_{d}\left(t_{j}, A_{j}\right)$. Thus, by (3.3.3) and (3.2), $\varphi_{d}\left(t_{0}, A_{0}\right) \subset L$. To prove $L \subset \varphi_{d}\left(t_{0}, A_{0}\right)$, let $x \in L$. Let $\delta>0$. Since $\left(t_{j}, A_{j}\right) \rightarrow\left(t_{0}, A_{0}\right)$ and $\varphi\left(t_{j}, A_{j}\right) \rightarrow L$ as $j \rightarrow \infty$, there exists $j_{0}$ such that

(1) $\left|t_{j_{0}}-t_{0}\right|<\delta / 3$,

(2) $H_{d}\left(A_{j_{0}}, A_{0}\right)<\delta / 3$, and

(3) $H_{d}\left[\varphi\left(t_{j_{0}}, A_{j_{0}}\right), L\right]<\delta / 3$.

Since $x \in L$, by (3) [and (2.6)] there exists $y \in \varphi\left(t_{j_{0}}, A_{j_{0}}\right)$ such that $d(x, y)<$ $\delta / 3$. Since $y \in \varphi_{d}\left(t_{j_{0}}, A_{j_{0}}\right)$, there exists $a_{j_{0}} \in A_{j_{0}}$ such that $d\left(y, a_{j_{0}}\right) \leqslant t_{j_{0}}$. Since 
$a_{j_{0}} \in A_{j_{0}}$, by (2) [and (2.6)] there exists $a_{0} \in A_{0}$ such that $d\left(a_{j_{0}}, a_{0}\right)<\delta / 3$. Hence,

$$
d\left(x, a_{0}\right) \leqslant d(x, y)+d\left(y, a_{j_{0}}\right)+d\left(a_{j_{0}}, a_{0}\right)<t_{j_{0}}+(2 \delta) / 3 .
$$

Thus, by (1),

$$
d\left(x, a_{0}\right)<t_{0}+\delta
$$

We have shown that

(4) given $\delta>0$, there exists a point $a(\delta) \in A_{0}$ such that $d(x, a(\delta))<t_{0}+$ $\delta$.

By using (4) and the compactness of $A_{0}$, it follows easily that there exists a point $a \in A_{0}$ such that $d(x, a) \leqslant t_{0}$. Hence, $x \in \varphi_{d}\left(t_{0}, A_{0}\right)$. Therefore, since $x \in L$ was arbitrary, $L \subset \varphi_{d}\left(t_{0}, A_{0}\right)$. Thus, we have proved (*) and, therefore, we have proved $\varphi_{d}$ is continuous at $\left(t_{0}, A_{0}\right)$. Hence, since $\left(t_{0}, A_{0}\right) \in[0,+\infty)$ $\times 2^{X}$ was arbitrary, $\varphi_{d}$ is continuous. Therefore, we have proved (3.3.3) implies (3.3.1). This completes the proof of (3.3).

The following corollary will be used in $\$ \$ 4$ and 5 .

(3.4) Corollary. If $(X, \rho)$ is a continuum with a convex metric $\rho$, then $\varphi_{\rho}$ [as defined in (3.1)] is continuous.

Proof. By (2.8), (3.3.2) holds with $d=\rho$. Hence, by (3.3), (3.3.1) holds for $\varphi_{\rho}$.

(3.5) Remark. Fraser [6, Theorem 1] has shown that (3.3.2) implies $X$ is a locally connected continuum. Hence, by (3.3), (3.3.1) implies $X$ is a locally connected continuum. By using [2, Theorem 8] or [10] to (possibly) change the metric, (3.4) gives a partial or "topological" converse.

4. Some lemmas. In (4.1), we define a function $\alpha_{d}$ and, in (4.2) and (4.5), we give two results about $\alpha_{d}$ which will be used in $\$ 5$.

(4.1) Definition. Let $(X, d)$ be a continuum. Define $\alpha_{d}: 2^{X} \rightarrow[0,+\infty)$ by: For each $A \in 2^{X}$,

$$
\alpha_{d}(A)=\inf \left\{t \geqslant 0: K_{d}(t, A) \text { is connected }\right\} .
$$

(4.2) Main Lemma 1. Let $(X, d)$ be a continuum. Then, for any $A \in 2^{X}$, $K_{d}\left(\alpha_{d}(A), A\right)$ is connected.

Proof. Let $A \in 2^{X}$. By definition of $\alpha_{d}(A)$, there exist numbers $t_{n}, n=$ $1,2, \ldots$, satisfying (1) through (3) below:

(1) $t_{n} \geqslant t_{n+1} \geqslant \alpha_{d}(A)$ for each $n=1,2, \ldots$;

(2) $K_{d}\left(t_{n}, A\right)$ is connected for each $n=1,2, \ldots$;

(3) $t_{n} \rightarrow \alpha_{d}(A)$ as $n \rightarrow \infty$.

By (1),

(4) $K_{d}\left(t_{n}, A\right) \supset K_{d}\left(t_{n+1}, A\right)$ for each $n=1,2, \ldots$

By (1), (3), and the compactness of $A$, it follows that

(5) $K_{d}\left(\alpha_{d}(A), A\right)=\cap_{n=1}^{\infty} K_{d}\left(t_{n}, A\right)$.

By (2) [and (2.5)], (4) and (5), we see that $K_{d}\left(\alpha_{d}(A), A\right)$ is a nested intersection 
of continua. Hence $\left[13,9.4\right.$, p. 15], $K_{d}\left(\alpha_{d}(A), A\right)$ is connected. This proves (4.2).

(4.3) LeMma. Let $(X, \rho)$ be a continuum with a convex metric $\rho$. Let $A \in 2^{X}$ and let $t \geqslant 0$. Then $K_{\rho}(t, A)$ is connected if and only if $t \geqslant \alpha_{\rho}(A)$.

Proof. Let $A \in 2^{X}$ and let $t \geqslant 0$. It follows easily from the definition in (4.1) that if $K_{\rho}(t, A)$ is connected (and $\left.t \geqslant 0\right)$, then $t \geqslant \alpha_{\rho}(A)$. To prove the converse, first note the following simple consequence of (2.4) and (2.7):

(i) If $x \in K_{\rho}(t, A)$, then there is a connected subset $\gamma_{x}$ of $K_{\rho}(t, A)$ such that $x \in \gamma_{x}$ and $A \cap \gamma_{x} \neq \varnothing$.

Now, assume $t \geqslant \alpha_{\rho}(A)$. Then, $K_{\rho}\left(\alpha_{\rho}(A), A\right) \subset K_{\rho}(t, A)$. Hence, by (4.2),

(2) $K_{\rho}\left(\alpha_{\rho}(A), A\right)$ is a connected subset of $K_{\rho}(t, A)$ such that, since $\alpha_{\rho}(A) \geqslant$ $0, A \subset K_{\rho}\left(\alpha_{\rho}(A), A\right)$.

It follows easily from (1) and (2) that $K_{\rho}(t, A)$ is connected. This completes the proof of (4.3).

(4.4) Lemma. Let $(X, \rho)$ be a continuum with a convex metric $\rho$. Let $A, B \in 2^{X}$. If $t \geqslant 0$ such that $K_{\rho}(t, A)$ is connected and if $\eta \geqslant H_{\rho}(A, B)$, then $K_{\rho}(t+\eta, B)$ is connected.

Proof. We will show that

$$
\begin{aligned}
& \text { if } x \in K_{\rho}(t+\eta, B), \text { then there is a connected subset } G_{x} \text { of } \\
& K_{\rho}(t+\eta, B) \text { such that } x \in G_{x} \text { and } A \cap G_{x} \neq \varnothing .
\end{aligned}
$$

To prove (*), let $x \in K_{\rho}(t+\eta, B)$. Then, there exists $b \in B$ such that $\rho(x, b) \leqslant t+\eta$. By (2.7), there exists $\gamma_{1} \subset X$ such that $x, b \in \gamma_{1}$ and such that $\gamma_{1}$ is isometric to $[0, \rho(x, b)]$. Since $H_{\rho}(A, B) \leqslant \eta$ and $b \in B$, there exists $a \in A$ such that $\rho(b, a) \leqslant \eta$. By (2.7), there exists $\gamma_{2} \subset X$ such that $b, a \in \gamma_{2}$ and such that $\gamma_{2}$ is isometric to $[0, \rho(b, a)]$. It is easy [using the isometries] to verify that $G_{x}=\gamma_{1} \cup \gamma_{2}$ satisfies (*). This completes the proof of (*). Since

$$
A \subset K_{\rho}(t, A) \subset K_{\rho}(t+\eta, B)
$$

and $K_{\rho}(t, A)$ is connected, it follows from (*) that $K_{\rho}(t+\eta, B)$ is connected. This completes the proof of (4.4).

(4.5) Main Lemma 2. Let $(X, \rho)$ be a continuum with a convex metric $\rho$. Then $\alpha_{\rho}: 2^{X} \rightarrow[0,+\infty)$ is continuous.

Proof. Let $\eta>0$. Let $L_{1}, L_{2} \in 2^{X}$ such that $H_{\rho}\left(L_{1}, L_{2}\right) \leqslant \eta$. By (4.3), $K_{\rho}\left(\alpha_{\rho}\left(L_{1}\right), L_{1}\right)$ is connected. Hence, by (4.4), $K_{\rho}\left(\alpha_{\rho}\left(L_{1}\right)+\eta, L_{2}\right)$ is connected. Hence, by (4.1),

$$
\alpha_{\rho}\left(L_{2}\right) \leqslant \alpha_{\rho}\left(L_{1}\right)+\eta \text {. }
$$

By interchanging the roles of $L_{1}$ and $L_{2}$ in the argument just given, we obtain

$$
\alpha_{\rho}\left(L_{1}\right) \leqslant \alpha_{\rho}\left(L_{2}\right)+\eta \text {. }
$$

Combining (1) and (2), we obtain 


$$
\left|\alpha_{\rho}\left(L_{1}\right)-\alpha_{\rho}\left(L_{2}\right)\right| \leqslant \eta
$$

From what we have shown, $\alpha_{\rho}$ is continuous and, therefore, we have proved (4.5).

5. Proof of (1.2). Assume $(X, d)$ is a continuum. To prove (1.2.1), assume $(X, d)$ is locally connected. Then, by [2, Theorem 8] or [10], there is a convex metric $\rho$ for $X$; i.e., $\rho$ is convex and $(X, \rho)$ is homeomorphic to $(X, d)$. Let $\varphi_{\rho}$ and $\alpha_{\rho}$ be as in (3.1) and (4.1) respectively. For each $A \in 2^{X}$, let $r(A)=$ $\varphi_{\rho}\left(\alpha_{\rho}(A), A\right)$. By (4.2) [and (2.5)], the formula above defines a function $r$ from $2^{X}$ to $C(X)$. By (3.4) and (4.5), it follows that $r$ is continuous. If $A \in C(X)$, then $\alpha_{\rho}(A)=0$ [by (4.1)] and, hence $r(A)=A$. Since $\alpha_{\rho}(A) \geqslant 0$ for each $A \in 2^{X}$ [by (4.1)], $r(A) \supset A$ for each $A \in 2^{X}$. Thus, $r$ has all the properties in (1.2.1) and, therefore, we have proved (1.2.1).

To prove (1.2.2), assume $g$ satisfies the hypotheses of (1.2.2). Let

$$
\Gamma=\left\{A \in 2^{X}: g(A) \supset A\right\}
$$

and let

$$
\Lambda=\left\{A \in 2^{X}: g(A) \subset A\right\}
$$

By hypothesis,

(1) $\Gamma$ and $\Lambda$ are closed subsets of $2^{X}$ such that $\Gamma \cup \Lambda=2^{X}$ and $\Gamma \cap \Lambda=$ $C(X)$.

We will take three cases.

Case 1. $\Gamma-C(X) \neq \varnothing \neq \Lambda-C(X)$. Then, it follows using (1) that $2^{X}-C(X)$ is not connected. This contradicts $(2.11)$.

Case 2. $\Gamma-C(X)=\varnothing$. Then, by (1), $\Lambda=2^{X}$. Hence, since (by hypothesis) $g(A) \in C(X)$ for each $A \in 2^{X}$, we see that $\left[F_{n}(X)\right.$ is defined in (2.10)].

(2) for each $A \in F_{2}(X), g(A) \in F_{1}(X)$ and the unique point in $g(A)$ is a point of $A$.

Let $g_{2}$ denote the restriction of $g$ to $F_{2}(X)$ and define $j: F_{1}(X) \rightarrow X$ by $j(\{x\})=x$ for each $\{x\} \in F_{1}(X)$. By (2), the function $j \circ g_{2}$ is defined on all of $F_{2}(X)$ and is a continuous selection. Hence, by [7, Theorem 1], $X$ is an arc.

Case 3. $\Lambda-C(X)=\varnothing$. Then, by (1), $\Gamma=2^{X}$. Hence, $g$ is a retraction from $2^{X}$ onto $C(X)$ such that $g(A) \supset A$ for each $A \in 2^{X}$. Using this, the proof given in [4] of the "only if" part of (1.1) above may be applied to see that $X$ is locally connected.

It follows from what we have done in Case 1 through Case 3 that $X$ is locally connected. Therefore, we have proved (1.2.2).

(5.1) REMARK. If $X$ is a locally connected continuum, then $C(X)$ is an absolute retract [14, pp. 190-191]. Thus, it was "known" that there is a retraction from $2^{X}$ onto $C(X)$ when $X$ is locally connected-(1.2.1) and its proof give a particular retraction with special properties. For example: Let $r$ be as defined in the proof of (1.2.1); then, it follows from the formula for $r$, 
(3.4), (4.5), and elementary properties of convex metrics that $r^{-1}(B)$ is contractible for each $B \in C(X)$. In particular, it can be shown that the formula

$$
h(A, s)=\varphi_{\rho}\left(s \cdot \alpha_{\rho}(A), A\right)
$$

defines a homotopy $h: r^{-1}(\mathrm{~B}) \times[0,1] \rightarrow r^{-1}(B)$ such that $h(A, 0)=A$ and $h(A, 1)=r(A)=B$ for each $A \in r^{-1}(B)$.

6. A hyperspace problem-concluding comments. In [11], I asked the following question:

(6.1) Question [11, 3.7, p. 194]. When is $C(X)$ a retract of $2^{X}$ ?

The investigations which led to (1.2) were originally motivated by (6.1). In particular, (1.2.2) indicates that a retraction from $2^{X}$ onto $C(X)$ must be rather complicated if $X$ is not locally connected. Recently, using the theory of semilattices to obtain some answers to (6.1), Lawson [8] has attempted to give some conditions which imply the existence of a retraction from $2^{X}$ onto $C(X)$. However, Jack Goodykoontz has pointed out to me that Lawson's paper contains an error. Hence, at the present time, there is no example of a nonlocally connected continuum $X$ for which it has been shown that there is a retraction from $2^{X}$ onto $C(X)$.

Many results in the literature suggested to me the possibility that $C(X)$ is always a retract of $2^{X}$. For example: [5, equivalence of (b) and (c) in (3.1)], [5, 3.4] and [12, Theorem 1.2], [3, Theorem 3], etc. Also in this connection, I mention that $C(X)$ is always a continuous image of $2^{X}[11,3.6]$. However, Jack Goodykoontz has recently found an example of a continuum $X$ such that $C(X)$ is not a retract of $2^{X}$. His paper, entitled " $C(X)$ is not necessarily a retract of $2^{X}$, , appears in this Proceedings.

\section{REFERENCES}

1. Nicholas Artemiadis, A remark on metric spaces, Nederl. Akad. Wetensch. Proc. Ser. A 68 (1965), 316-318.

2. R. H. Bing, Partitioning a set, Bull. Amer. Math. Soc. 55 (1949), 1101-1110.

3. Jack T. Goodykoontz, Jr., Connectedness im kleinen and local connectedness in $2^{X}$ and $C(X)$, Pacific J. Math. 53 (1974), 387-397.

4. Some functions on hyperspaces of hereditarily unicoherent continua, Fund. Math. 95 (1977), 1-10.

5. J. L. Kelley, Hyperspaces of a continuum, Trans. Amer. Math. Soc. 52 (1942), 22-36.

6. Robert B. Fraser, A new characterization of Peano continua, Prace Mat. 16 (1972), 247-248.

7. K. Kuratowski, S. B. Nadler, Jr. and G. S. Young, Continuous selections on locally compact separable metric spaces, Bull. Acad. Polon. Sci. 18 (1970), 5-11.

8. J. D. Lawson, Applications of topological algebra to hyperspace problems, Proc. Memphis State University Topology Conference (to appear).

9. K. Menger, Untersuchungen über allgemeine Metrik, Math. Ann. 100 (1928), 75-163.

10. E. E. Moise, Grille decomposition and convexification theorems for compact metric locally connected continua, Bull. Amer. Math. Soc. 55 (1949), 1111-1121.

11. Sam B. Nadler, Jr., Some problems concerning hyperspaces, Topology Conference (V.P.I. and S.U.), Lecture Notes in Math., vol. 375, Springer-Verlag, New York, 1974, pp. 190-197.

12. J. Segal, Hyperspaces of the inverse limit space, Proc. Amer. Math. Soc. 10 (1959), 706-709. 
13. Gordon Thomas Whyburn, Analytic Topology, Amer. Math. Soc. Colloq. Publ., vol. 28, Amer. Math. Soc., Providence, R.I., 1942.

14. M. Wojdyslawski, Rétractes absolus et hyperespaces des continus, Fund. Math. 32 (1939), 184-192.

DePartment of Mathematics, UnIVERSTTY of KentuCKy, LeXINGTON, KeNTUCKY 40506

Current address (S. B. Nadler, Jr.): Department of Mathematics, University of Saskatchewan, Saskatoon, Canada S7N OW0 